\title{
Esophageal Variceal Bleeding in Hospitalized Patients With Cirrhosis
}

\author{
Chaitanya Pant, MD¹, Madhav Desai, MD, MPH¹, Abhishek Deshpande, MD, PhD2,3, Ryan Taylor, MD¹, \\ Mojtaba Olyaee, MD¹, Richard Gilroy, MD*
}

\begin{abstract}
${ }^{1}$ Division of Gastroenterology, Hepatology and Motility, Department of Internal Medicine, University of Kansas Medical Center, Kansas City, Kansas; ${ }^{2}$ Medicine Institute Center for Value Based Care Research, Cleveland Clinic, Cleveland, Ohio; ${ }^{3}$ Department of Infectious Diseases, Medicine Institute, Cleveland Clinic, Cleveland, Ohio.
\end{abstract}

Esophageal variceal bleeding (EVB) is a frequent complication in cirrhotic patients resulting in considerable mortality and morbidity. The aim of this study was to investigate the occurrence, impact, and trends of EVB in hospitalized cirrhotic patients on a nationwide level in the United States. We interrogated data from the Nationwide Inpatient Sample from 2002 to 2012. Utilizing International Classification of Diseases, Ninth Revision, Clinical Modification codes, we analyzed hospital discharges for cirrhosis and related EVB in adult patients. EVB in cirrhotic patients was independently associated with overall worse outcomes with respect to in-hospital mortality (10\% vs $5 \% ; P<0.01$ ) and hospital charges (median $\$ 41,000$ vs $\$ 26,000 ; P<0.01$ ). In the period from 2002 to 2012, the number of cirrhosis-related hospitalizations increased from 337,956 to 570,220 $(P<0.01)$. Concurrently, the incidence of EVB in hospitalized cirrhotic patients declined from $8.60 \%$ to $5.78 \%$, with an overall decreased trend $(P<0.01)$. The decline in the rate of EVB in hospitalized cirrhotic patients from 2002 to 2012 likely reflects the effectiveness of primary and secondary prophylaxis. Journal of Hospital Medicine 2015;10:453-456. (C) 2015 Society of Hospital Medicine
Cirrhosis is a leading cause of death in the United States. In 2010, cirrhosis resulted in an estimated 49,500 deaths, which represented a significant increase from 35,500 deaths 2 decades ago. ${ }^{1}$ Cirrhotic patients are susceptible to numerous disease-specific complications including ascites, esophageal varices, hepatic encephalopathy (HE), and hepatorenal syndrome (HRS). ${ }^{2}$

Esophageal varices develop in approximately 50\% of patient with cirrhosis, and their presence correlates with the severity of liver disease. ${ }^{3}$ In cirrhotic patients, esophageal variceal bleeding (EVB) occurs at an annual rate of $5 \%$ to $15 \%$ and results in substantial morbidity and mortality. ${ }^{3}$ Utilizing US national data, Jamal et al. reported a decline in the rate of hospitalizations related to EVB from 1988 to $2002 .{ }^{4}$ However, recent large-scale studies relating to the epidemiology of EVB are lacking. We conducted a retrospective analysis using a national US database to study the differences in demographic characteristics, rate of complications, outcomes, and temporal trends in hospitalized cirrhotic patients with and without EVB.

\footnotetext{
*Address for correspondence and reprint requests: Richard Gilroy, MD, Division of Gastroenterology, Hepatology, \& Motility, Department of Internal Medicine, University of Kansas Medical Center, 3901 Rainbow Boulevard, Kansas City, KS 66160; Telephone: 913-588-5000; Fax: 913-588-8390.

E-mail: rgilroy15@gmail.com

Additional Supporting Information may be found in the online version of this article.

Received: December 26, 2014; Revised: March 4, 2015; Accepted: March 9, 2015

2015 Society of Hospital Medicine DOI 10.1002/jhm.2360

Published online in Wiley Online Library (Wileyonlinelibrary.com).
}

\section{METHODS}

We utilized biennial data (2002-2012) from the Healthcare Cost and Utilization Project Nationwide Inpatient Sample using methods described earlier. ${ }^{5}$ Initially, we extracted all entries with any discharge diagnosis of cirrhosis (International Classification of Diseases, Ninth Revision, Clinical Modification [ICD9-CM] codes: 571.2, 571.5, 571.6) in adult patients ages 18 years and older. ${ }^{6}$ Within this cirrhotic population, we next extracted all entries with any discharge diagnosis of EVB (ICD-9-CM codes: 456.0., 456.20). ${ }^{6}$ Population-based rates relating to hospital discharges were reported as per 100,000 population/year.

The outcome variables of interest were in-hospital mortality, total charges (rounded to the nearest $\$ 1000$ ) and length of stay (LOS). Demographic details and hospital characteristics were also extracted. Cases were queried for complications well recognized in cirrhotic patients. These included urinary tract infection (UTI) (ICD-9-CM codes: 1122, 59010-11, 5902-03, 59080-81, 5950, 5970, 5990), skin and subcutaneous tissue infections (SSCI) (ICD-9-CM codes: 680-82, 684, 686), spontaneous bacterial peritonitis (SBP) (ICD-9-CM codes: 56723, 5672), Clostridium difficile infection (ICD-9-CM code: 00845), or pneumonia (ICD-9-CM codes: 480-83, 487). ${ }^{6}$ Also queried were HE (ICD-9-CM code: 572.2$)^{7}$ and HRS (ICD-9-CM code: 572.4). ${ }^{8}$ Comorbid conditions were assessed using the Elixhauser comorbidity index minus the presence of liver disorders but including alcohol abuse. $^{9}$

Statistical analyses were performed using SAS version 9.3 (SAS Institute, Cary, NC). To determine the independent association of EVB on outcome variables, we performed case-control matching (EVB vs no EVB). We used high-dimensional propensity scores in 
TABLE 1. Sociodemographic Factors, Deaths, Complications, and Outcome Indicators of Hospitalized Cirrhotic Patients With and Without Esophageal Variceal Bleeding

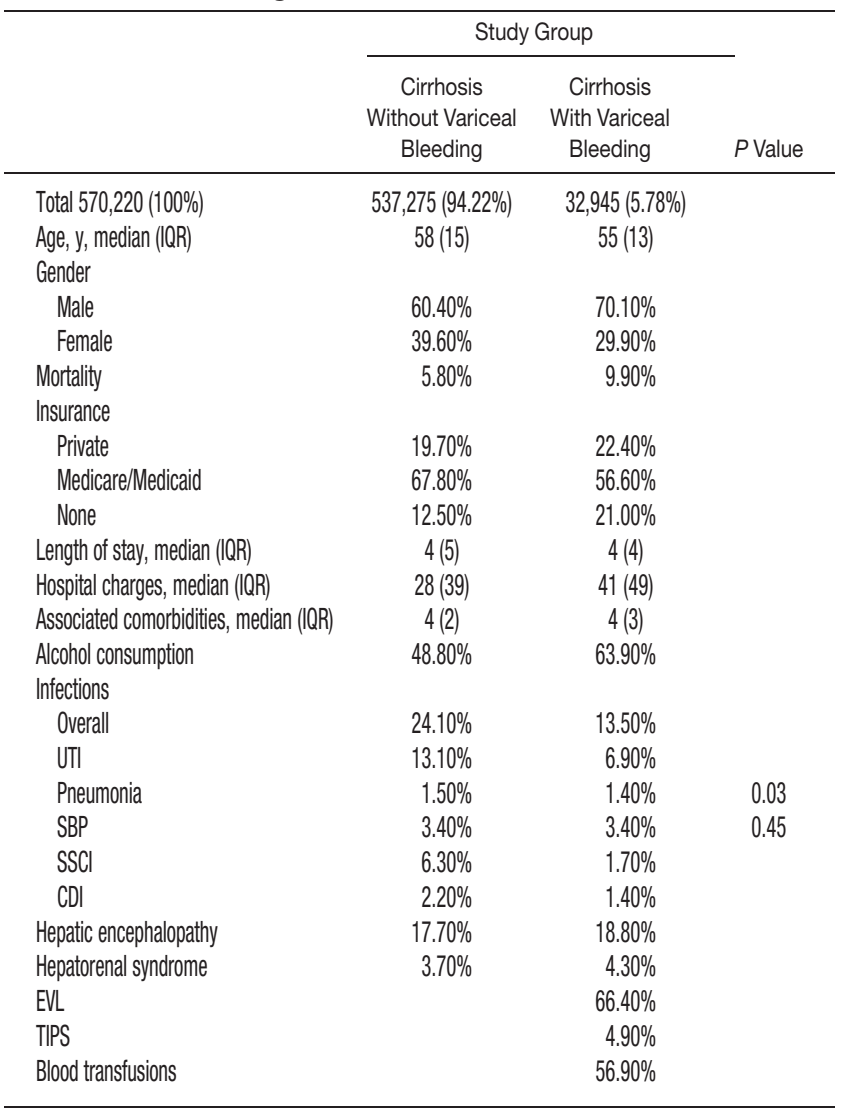

NOTE: Weighted data from Healthcare Cost and Utilization Project Nationwide Inpatient Sample for the year 2012. All comparisons significant at $P<0.01$ unless indicated otherwise. Abbreviations: CDI, Clostridium difficile infection; EVL, endoscopic variceal ligation; IQR, interquartile range; SBP, spontaneous bacterial peritonitis; SSCl, skin and subcutaneous tissue infections; TIPS, transjugular intrahepatic portosystemic shunt; UTI, urinary tract infections.

a 1:5 matching ratio with a greedy matching algorithm generated by regression analysis of patients with EVB based on demographics details (age, gender, insurance status), comorbid conditions, alcohol abuse, infections as detailed above, HE, and HRS. The $\chi^{2}$ test and the Mann-Whitney $U$ test compared categorical and continuous variables. For trend analysis, we used the Cochrane-Armitage test. The threshold for significance for all analyses was $P<0.01$.

\section{RESULTS}

In 2012, there were 570,020 hospital discharges related to cirrhosis in patients 18 years of age and older. Within this cohort, EVB occurred in 32,945 discharges $(5.78 \%)$. Table 1 details differences between cirrhotic patients with and without EVB. Comparatively, patients with EVB were younger (median age 55 years, interquartile range [IQR] 13 years vs median age 58 years, IQR 15 years; $P<0.01$ ), more likely to be male $(70.1 \%$ vs $60.4 \% ; P<0.01)$, and without health insurance $(21.0 \%$ vs $12.50 \% ; P<0.01)$. Minor

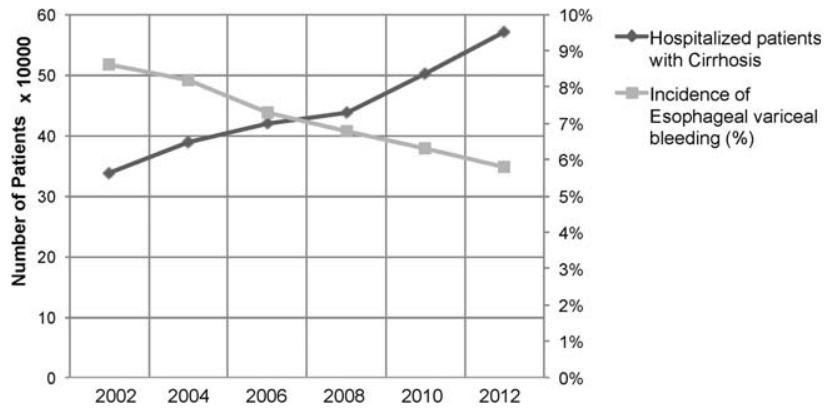

FIG. 1. Temporal trends of hospital discharges with diagnosis of cirrhosis and incidence of esophageal variceal bleeding in this population. Weighted data from the Healthcare Cost and Utilization Project Nationwide Inpatient Sample for the years 2002 to 2012.

differences between the 2 groups were observed in respect to hospital region, location, teaching status, and household income quartile. There was no difference in the number of comorbid conditions (median 4 comorbid conditions in each group).

Patients with EVB suffered a significantly higher rate of alcohol abuse $(63.90 \%$ vs $48.80 \%$; $P<0.01)$. EVB was also associated with an overall lower incidence of infection $(13.50 \%$ vs $24.10 \% ; P<0.01)$. Specifically, the greatest difference in rates of infection were observed for UTI $(6.90 \%$ vs $13.10 \% ; P<0.01)$ and SSCI $(1.70 \%$ vs $6.30 \% ; P<0.01)$. Also, patients with EVB demonstrated a small, yet significant increased incidence of $\mathrm{HE} \quad(18.80 \%$ vs $17.70 \%$; $P<0.01)$ and HRS $(4.30 \%$ vs $3.70 \% ; P<0.01)$.

Cirrhotic patients with EVB demonstrated worse overall outcomes compared to their counterparts without EVB. This manifested in an unadjusted higher mortality rate $(9.90 \%$ vs $5.80 \% ; P<0.01)$ and increased hospital charges (median $\$ 41,000$ [IQR $\$ 49,000$ ] vs $\$ 28,000$ [IQR $\$ 39,000] ; P<0.01)$. LOS between the 2 groups did not differ (median 4 days). After adjusting for demographic differences, complications, and comorbid conditions, EVB in patients with cirrhosis continued to be independently associated with a higher mortality rate $(10.00 \%$ vs $5.00 \%$; $P<0.01$ ) and increased hospital charges (median $\$ 41,000$ [IQR $\$ 49,000]$ vs $\$ 26,000$ [IQR $\$ 34,000$ ]; $P<0.01)$. Again, LOS was similar for the 2 groups (median 4 days).

Between the years 2002 and 2012, the number of hospital discharges related to cirrhosis increased from 337,956 to $570,220(P<0.01)$. Concurrently, the incidence of EVB in this population declined from $8.60 \%$ to $5.78 \%$ (Figure 1), representing an overall decrease of $33.0 \%$ with a significant decreased trend $(P<0.01)$.

We also calculated population-adjusted hospitalization rates for discharges related to cirrhosis and EVB. The rate of cirrhosis-related discharges continued to demonstrate an increased trend from 157.42/100,000 population in 2002 to $237.43 / 100,000$ population in $2012(P<0.01)$. However, no significant trend was 
observed for EVB-related hospital discharges in the same period of time $(13.60 / 100,000$ population in 2002 to $13.72 / 100,000$ population in 2012; $P=0.91$ ).

\section{DISCUSSION}

Our results indicated a significantly higher rate of alcohol abuse in cirrhotic patients with EVB. Alcohol consumption is an independent risk factor for esophageal variceal bleeding. ${ }^{10,11}$ Continued alcohol consumption not only increases the risk for development of varices but may also precipitate variceal rupture. ${ }^{10}$ Other risk factors associated with EVB in this study (younger age, male, lower economic status) are likely related to a higher incidence of alcohol abuse in this demographic. ${ }^{12}$

Patients with EVB were also noted to have a lower overall incidence of infection, especially UTI and SSCI. The use of broad-spectrum antibiotics decreases mortality from secondary infection and improves the prognosis of cirrhotic patients with EVB. ${ }^{13,14}$ The American Association for the Study of Liver Diseases recommends the use of third-generation cephalosporins in the setting of EVB. ${ }^{3}$ The widespread adoption of this in clinical practice may have contributed to a decreased rate of infection in patients with EVB. The difference in the incidence rates of HE and HRS, although statistically significant, were small, and likely the consequence of the large numbers involved in our study.

Our results also indicate that cirrhotic patients with EVB were twice as likely to die compared to matched counterparts without EVB. The increased mortality associated with EVB could be related to hemorrhagic/ hypovolemic shock and cardiovascular collapse, aspiration into airway, multiorgan dysfunction due to poor perfusion, infections including SBP, and HE. Although prior studies have demonstrated the relationship between EVB and increased mortality, typically they have been restricted to small single-center studies involving fewer than 200 patients. ${ }^{6-9}$ Cirrhotic patients with EVB also incurred significantly higher hospital charges compared to matched counterparts. Interestingly, the hospital LOS did not differ between the 2 groups. Intensive care and procedural costs were likely a major contributor to the higher charges; cirrhotic patients with EVB underwent a median of 3 procedures (IQR 2) during their hospital stay compared to a median of 1 procedure (IQR 3) for cirrhotic patients without EVB $(P<0.01$; data not shown).

In contrast to trends from earlier decades, ${ }^{4}$ the population-adjusted rate of EVB-related hospital discharges did not change significantly from 2002 to 2012. However, these data are confounded in their interpretation by a substantial increase in the prevalence of cirrhosis in the United States during the same time period. ${ }^{15}$ Therefore, it may be more meaningful to state that there was a contemporaneous decline in
EVB-related hospital discharges when considered in the context of a complicating rate in hospitalized cirrhotic patients. These results are consistent with a recent single-center study ${ }^{16}$ and are very likely the fruition of intensive screening programs with primary and secondary prophylaxis for EVB involving esophageal variceal ligation and pharmacotherapy $(\beta$-blockers) as well as the increased acceptance of transjugular intrahepatic portosystemic shunt placement. ${ }^{17-19}$

There are limitations to our study. First, we relied exclusively on ICD-9-CM codes for case identification. Second, there is a nonavailability of data pertaining to Model for End-Stage Liver Disease score calculations, medication, and antibiotic usage. Third, the Nationwide Inpatient Sample database does not allow for distinguishing individual patients with repeat admissions. Finally, our results represent a weighted estimate of national data.

\section{CONCLUSION}

EVB in cirrhotic patients was associated with significantly higher mortality and increased hospital charges. Also, the rate of EVB-related hospital discharges as a complicating factor in patients with cirrhosis declined significantly during the decade 2002 to 2012. This likely reflects the ongoing effectiveness of primary and secondary prophylaxis.

\section{Acknowledgements}

The authors acknowledge the Healthcare Cost and Utilization Project (HCUP) Nationwide Inpatient Sample, sponsored by the Agency for Healthcare Research and Quality, which contributes to HCUP (http:// www.hcup-us.ahrq.gov/db/hcupdatapartners.jsp).

Disclosures: C.P., the first author and corresponding author, conceptualized the study, and with A.D. gathered and analyzed the data. C.P. and M.D. wrote, edited, and proofread the manuscript as well as created the bibliography and formulated the table and figure. R.G., R.T., and M.O. edited, commented on, and reviewed the manuscript. All of the authors reviewed and agreed on the final version of the manuscript for submission. The authors report no conflicts of interest.

\section{References}

1. US Burden of Disease Collaborators. The state of US health, 19902010: burden of diseases, injuries, and risk factors. JAMA. 2013; 310(6):591-608.

2. Rahimi RS, Rockey DC. Complications of cirrhosis. Curr Opin Gastroenterol. 2012;28(3):223-229.

3. Garcia-Tsao G, Sanyal AJ, Grace ND, Carey W, Practice Guidelines Committee of the American Association for the Study of Liver Diseases, Practice Parameters Committee of the American College of Gastroenterology. Prevention and management of gastroesophageal varices and variceal hemorrhage in cirrhosis. Hepatology. 2007;46(3): 922-938.

4. Jamal MM, Samarasena JB, Hashemzadeh M, Vega KJ. Declining hospitalization rate of esophageal variceal bleeding in the United States. Clin Gastroenterol Hepatol. 2008;6(6):689-695; quiz 605.

5. Pant C, Anderson MP, O'Connor JA, Marshall CM, Deshpande A, Sferra TJ. Association of Clostridium difficile infection with outcomes of hospitalized solid organ transplant recipients: results from the 2009 Nationwide Inpatient Sample database. Transpl Infect Dis. 2012; 14(5):540-547.

6. Singal AK, Salameh H, Kamath PS. Prevalence and in-hospital mortality trends of infections among patients with cirrhosis: a nationwide study of hospitalised patients in the United States. Aliment Pharmacol Ther. 2014;40(1):105-112.

7. Nguyen GC, Segev DL, Thuluvath PJ. Racial disparities in the management of hospitalized patients with cirrhosis and complications of portal hypertension: a national study. Hepatology. 2007;45(5):12821289. 
8. Sam J, Nguyen GC. Protein-calorie malnutrition as a prognostic indicator of mortality among patients hospitalized with cirrhosis and portal hypertension. Liver Int. 2009;29(9):1396-1402.

9. Elixhauser A, Steiner C, Harris DR, Coffey RM. Comorbidity measures for use with administrative data. Med Care. 1998;36(1):8-27.

10. Liao WC, Hou MC, Chang CJ, Lee FY, Lin HC, Lee SD. Potential precipitating factors of esophageal variceal bleeding: a case-control study. Am J Gastroenterol. 2011;106(1):96-103.

11. Luca A, Garcia-Pagan JC, Bosch J, et al. Effects of ethanol consumption on hepatic hemodynamics in patients with alcoholic cirrhosis. Gastroenterology. 1997;112(4):1284-1289.

12. Hasin DS, Stinson FS, Ogburn E, Grant BF. Prevalence, correlates, disability, and comorbidity of DSM-IV alcohol abuse and dependence in the United States: results from the National Epidemiologic Survey on Alcohol and Related Conditions. Arch Gen Psychiatry. 2007;64(7): 830-842.

13. Lee YY, Tee HP, Mahadeva S. Role of prophylactic antibiotics in cirrhotic patients with variceal bleeding. World J Gastroenterol. 2014; 20(7):1790-1796.
14. Chavez-Tapia NC, Barrientos-Gutierrez T, Tellez-Avila F, et al. Metaanalysis: antibiotic prophylaxis for cirrhotic patients with upper gastrointestinal bleeding - an updated Cochrane review. Aliment Pharmacol Ther. 2011;34(5):509-518.

15. Scaglione S, Kliethermes S, Cao G, et al. The epidemiology of cirrhosis in the United States: a population-based study [published online ahead of print October 8, 2014]. J Clin Gastroenterol. doi: 10.1097/ MCG.0000000000000208.

16. Lim N, Desarno MJ, Lidofsky SD, Ganguly E. Hospitalization for variceal hemorrhage in an era with more prevalent cirrhosis. World $J$ Gastroenterol. 2014;20(32):11326-11332.

17. Gluud LL, Krag A. Banding ligation versus beta-blockers for primary prevention in oesophageal varices in adults. Cochrane Database Syst Rev. 2012;8:CD004544.

18. Garcia-Pagan JC, Caca K, Bureau C, et al. Early use of TIPS in patients with cirrhosis and variceal bleeding. N Engl J Med. 2010; 362(25):2370-2379.

19. Thiele M, Krag A, Rohde U, Gluud LL. Meta-analysis: banding ligation and medical interventions for the prevention of rebleeding from oesophageal varices. Aliment Pharmacol Ther. 2012;35(10):1155-1165. 